\title{
Server-Based Enterprise Collaboration Software Improves Safety and Quality in High-Volume PET/CT Practice
}

\author{
James E. McDonald, Marcus M. Kessler, Jeremy L. Hightower, CNMT, RT(CT), Susan D. Henry, and Linda A. Deloney \\ Division of Nuclear Medicine, Department of Radiology, University of Arkansas for Medical Sciences, Little Rock, Arkansas
}

\begin{abstract}
With increasing volumes of complex imaging cases and rising economic pressure on physician staffing, timely reporting will become progressively challenging. Current and planned iterations of PACS and electronic medical record systems do not offer workflow management tools to coordinate delivery of imaging interpretations with the needs of the patient and ordering physician. The adoption of a server-based enterprise collaboration software system by our Division of Nuclear Medicine has significantly improved our efficiency and quality of service.
\end{abstract}

Key Words: patient safety; quality assurance; practice management

J Nucl Med Technol 2013; 41:289-291

DOI: $10.2967 /$ jnmt.113.128033

\section{A} significant component of the contribution of imaging to high-quality care depends on timely delivery of results. With imaging examinations increasing in the coming years as economic constraints on physician staffing accelerate, timely interpretation will become more challenging. This will especially be the case with reports of complex modalities such as PET/CT that are disproportionately time-consuming to produce but essential for clinical decision making.

We performed and interpreted more than 6,500 PET/CT examinations in 2011, with volume up $10 \%$ year-over-year compared with 2010. This increased volume, together with a decrease in our number of faculty members in 2011, rendered inadequate the old approach of trying to keep pace by selecting the next examination "off the top of the stack."

Because we had no means of prioritizing examinations in order of need, time was spent on interpretations of studies on patients not scheduled to see the referring physician for days or even weeks at the expense of interpretations required the same or next day. As we fell behind clinical demand, calls for reports and enquiries about examination status increased. Considerable faculty time was wasted

Received Jun. 21, 2013; revision accepted Aug. 14, 2013.

For correspondence or reprints contact: James E. McDonald, Division of Nuclear Medicine, Department of Radiology, University of Arkansas for Medical Sciences, Slot 556, 4301 W. Markham St., Little Rock, AR 72205.

E-mail: jemcdonald@uams.edu

Published online Oct. 24, 2013.

COPYRIGHT (C) 2013 by the Society of Nuclear Medicine and Molecular Imaging, Inc. each day in responding to the resulting interruptions while the satisfaction of patients and referring clinics with our service declined. Adoption of a method for prioritization of interpretation order and communication of examination status to all interested parties was imperative.

\section{MATERIALS AND METHODS}

A secure, Health Insurance Portability and Accountability Act (HIPAA)-compliant SharePoint (Microsoft Corp.; http://sharepoint. microsoft.com) calendar system was provided by the Information Technology Department of the University of Arkansas for Medical Sciences. Beginning in June 2012, we began development of a calendar system listing each patient with return visit time, pertinent examination data including injection site and dose, and availability of images for review. The system was implemented in July 2012.

Physicians and staff are granted appropriate access through an emailed invitation to a site on the enterprise SharePoint homepage entitled "Nuclear Medicine." Calendar data can be viewed in several formats. Best for clinical schedule management is an available spreadsheet-style format, ours being entitled "Jeremy's View" (referring to a coauthor, Jeremy Hightower), with each row containing the data for a particular PET/CT examination (Fig. 1).

The SharePoint platform enables graduated permission levels for modification and review of calendar entries. The authors retain full permission levels allowing addition and deletion of patient entries. Others are assigned modification and review permission in keeping with their responsibilities. Technologists can enter data for individual patients as tasks are completed. Referring physicians and their office personnel are granted read-only access. Because all modifications are available for viewing by all concerned, communication about examination status and progress is facilitated. HIPAA compliance is ensured by the institution's information technology procedures, and access is granted only to those individuals enrolled in the university's compliance program.

When an examination is scheduled, clerical staff members enter the patient name, identification number, and return oncology clinic appointment time. At the time of dose administration, technologists enter pertinent data including injection site and serum glucose level, thereby making this information available for entry into the report. Technologists enter the date of study completion in the designated cell in the examination's row, thereby signaling that Digital Imaging and Communications in Medicine data are available for retrieval from the PACS system for importation onto a physician workstation for fusion and interpretation. Technologists can also note additional information, including reasons for departure from protocol or examination cancellation. 


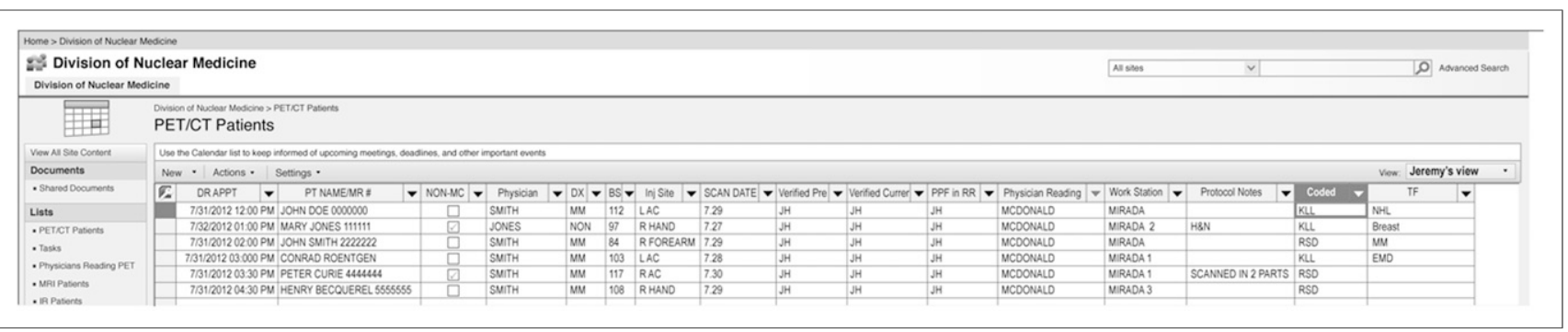

FIGURE 1. Example of “Jeremy's View” spreadsheet-style format for SharePoint homepage.

When selecting a case to read, nuclear medicine faculty members consult the calendar and choose from the completed studies of patient whose return visit to the clinic is most imminent. We indicate that we have taken up a case by making an entry in the cell in the "Physician Reading" column for that patient's examination. When referring physicians wish to discuss the clinical findings for a case, the staff can immediately determine the nuclear medicine physician whom they should see. All authorized personnel in the referring clinics have been given access, allowing them to track examination status in real time.

With experience we have developed a set of rules to ensure that no case goes beyond an acceptable period before being interpreted, even if the return visit is not imminent. No examination is allowed to wait more than 3 working days for interpretation. Examinations on inpatients are read within $24 \mathrm{~h}$ of completion, regardless of the day of the week. Examinations on new patients are interpreted by the end of the first regularly scheduled workday after completion. Cases that meet one of these criteria are assigned a 5 PM slot for the required day, indicating to all that the patient's return clinic visit is later but that interpretation will be completed by the end of that workday.

\section{RESULTS}

In our Nuclear Medicine Division, the high number of requests for $\mathrm{PET} / \mathrm{CT}$ that we receive requires that we operate 2 scanners from 12-15 h per day and a single scanner on Saturday. Because so many of our cases are generated outside regular working hours, our goal must be on-time delivery of interpretations. From January through June 2012, before adoption of the new system, between 3 and 7 cases per day were not signed and available before the scheduled return visit. We received 6-8 calls per day from clinic staff seeking reports not yet generated. From July through November 2012, after adoption of the system, we missed our goal approximately once per day, usually because of inaccurate information received at the time that data were entered into the system.

Results are available to the referring physician by the time needed in approximately $95 \%$ of cases, compared with approximately $80 \%$ before adoption.

Compared with the period before implementation, the number of calls we received from clinic staff was reduced by $80 \%$.

\section{DISCUSSION}

When the need for a system to better manage workflow became apparent, we considered several. Web-based systems such as Google Calendar and other online calendars were not acceptable because their use was prohibited by HIPAA compliance concerns. The institution's Outlook Calendar (Microsoft Corp.) was secure but did not support the level of detail we required for individual entries. Our Sectra PACS system (Southeast Imaging) has no provision for correlation of imaging work lists with referring clinic workflow. Our institution is in the process of implementing an electronic medical record system (Epic Systems Corp.). Although highly sophisticated and in widespread use in academic medical centers throughout the country, its current iteration does not offer workflow management that coordinates interpretations with patients' return visits to the ordering physician.

SharePoint is an enterprise server application designed to facilitate collaboration, provide shared management of content, assist in the implementation of business processes, and provide access to information relevant to organizational goals. Shared information is presented in the form of Web pages accessible via a Web browser and maintained inhouse (1). This paper is, to our knowledge (on the basis of a PubMed search), the first published use of SharePoint to assist in management of workflow in an imaging practice.

Information on the progress of an examination is now available simultaneously and in real time to technologists, nuclear medicine faculty, and referring clinical staff. Interpreting physicians can see at a glance the status of a day's work flow, identify the most pressing cases, and indicate the completion of an examination. Staff from referring clinics can readily ascertain the status of their patients' examinations. They are encouraged to alert the nuclear medicine staff when the status of one of their patients unexpectedly changes, thus facilitating a 2-way flow of information that enhances efficiency and safety.

\section{CONCLUSION}

The adoption of a SharePoint calendar system by our Division of Nuclear Medicine has significantly improved efficiency, allowing us to improve service as PET/CT volumes increased and physician staffing declined. Although difficult to quantitate, a significant reduction in reading-room stress has resulted from adoption of the system as calls from referring-physician clinic staff have decreased. We believe referring-physician satisfaction has increased as well, as almost all reports are now available in a timely fashion. By granting read-only access to authorized personnel 
in the referring clinics, we believe we have boosted confidence in the efficiency of the PET/CT service and provided the staff of our key referring clinics with a sense of participation in what was previously for them a frustratingly opaque process. By granting access (at a task-appropriate level) to everyone involved with PET/CT, we are fostering an environment in which all stakeholders are given the means to help us improve the way we care for patients.

In addition to its clinical applicability, our experience has implications for future opportunities to improve the quality of processes. Nuclear medicine physicians and radiologists have succeeded by being early adopters of leading-edge imaging technologies. Sophisticated, Web-based applications to support business creativity and collaboration are rapidly developing and evolving. By adapting the skills we have developed to the application of data management and other tools for business enterprises, we may discover additional innovative ways to improve care.

\section{DISCLOSURE}

No potential conflict of interest relevant to this article was reported.

\section{REFERENCE}

1. Harvey G. SharePoint 2007 Collaboration for Dummies. Hoboken, NJ: Wiley Publishing, Inc.; 2009:10-13. 